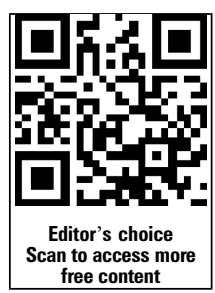

Department of Neurology, MS Center ErasMS, Erasmus MC University Medical Center, Rotterdam, The Netherlands

\section{Correspondence to}

T F Runia, Department of Neurology, MS Center ErasMS, Erasmus MC University Medical Center, Kamer Ee2230 faculteit, Postbus 2040, Rotterdam 3000 CA, The Netherlands:

t.runia@erasmusmc.nl

Received 21 April 2014 Revised 26 June 2014 Accepted 29 June 2014 Published Online First 22 July 2014
CrossMark

\footnotetext{
To cite: Runia TF, Jafari N, Siepman DAM, et al. J Neurol Neurosurg Psychiatry 2015;86:543-546.
}

\title{
Fatigue at time of CIS is an independent predictor of a subsequent diagnosis of multiple sclerosis
}

\author{
Tessel F Runia, Naghmeh Jafari, Dorine A M Siepman, Rogier Q Hintzen
}

\section{ABSTRACT}

Objective Fatigue is a common, disabling symptom of multiple sclerosis (MS), but little is known about fatigue in patients with clinically isolated syndrome (CIS), often the presenting symptom of MS. We aimed to investigate the prevalence and severity of fatigue in patients with $\mathrm{CIS}$, and its association with a diagnosis of clinically definite MS (CDMS).

Methods 127 patients were consecutively included in our ongoing prospective CIS study. At baseline, clinical, demographic, laboratory and MRI data were collected, and fatigue severity was assessed using Krupp's Fatigue Severity Scale (FSS); fatigue was defined as FSS $\geq 5.0$. Fatigue scores were compared with scores of 113 healthy controls and with scores from the literature. The association of fatigue with CDMS was calculated using Cox regression models.

Results The mean FSS of patients with CIS was 4.3, similar to MS patients, and significantly higher than that of healthy individuals $(p<0.001)$. Fatigue prevalence in patients with CIS $(46.5 \%)$ was significantly higher than in controls $(p<0.001)$. Fifty-two patients $(40.9 \%)$ reached CDMS during follow-up. Fatigue was associated with a diagnosis of CDMS in univariate analysis (HR 2.6, $95 \% \mathrm{Cl} 1.5$ to 4.6$)$ and in multivariate analysis correcting for sex, age, neuroanatomical localisation of CIS, 25-OH-vitamin D, anxiety, depression, MRI dissemination in space and gadolinium enhancement (HR 4.5, 95\% Cl 1.9 to 10.6).

Conclusions Already at the stage of CIS, fatigue is a very common symptom, with a severity similar to fatigue in MS patients. This fatigue seems unrelated to the type or severity of the attack. Importantly, we found that fatigue was an independent predictor of a subsequent diagnosis of MS.

\section{INTRODUCTION}

Fatigue is a very common and disabling symptom in patients with multiple sclerosis (MS), reported by over $75 \%$ of MS patients at some point in the course of the disease. ${ }^{1-3}$ Nevertheless, the mechanism of fatigue in MS patients remains poorly understood. The proposed causes range from altered cerebral activation or influences and effects of pro-inflammatory cytokines to sleep disorders or depression. $^{4}$ Even less is known about fatigue among patients with a clinically isolated syndrome (CIS), often the presenting symptom of MS.

In this study, we aimed to investigate the prevalence and severity of fatigue in patients with CIS, and its association with a diagnosis of clinically definite MS (CDMS).
Low vitamin $\mathrm{D}$ has been associated with fatigue in several conditions, such as systemic lupus erythematosus (SLE) $)^{5}$ and traumatic brain injury, ${ }^{7}$ and is considered to be involved in the development and disease course of MS. ${ }^{8-10}$ Therefore, a second aim of this study was to investigate if fatigue was associated with the vitamin D status of patients with CIS.

To do this, fatigue, 25-OH-D concentrations (the metabolite best reflecting vitamin D status) ${ }^{11}$ and other clinical parameters were measured in our ongoing prospective multicentre CIS study.

\section{METHODS}

\section{Participants}

Patients were consecutively included in our ongoing prospective CIS study, the PROUD study (Predicting the Outcome of a Demyelinating event). This observational study is a multicentre study of our tertiary referral centre for MS in collaboration with several regional hospitals. Patients with CIS suggestive of MS are included if they give informed consent and fulfil the following inclusion criteria: age between 18 and 50 years, inclusion within 6 months after symptom onset and no serious comorbidity. At baseline, clinical and demographic data are collected, an MRI is performed and fatigue is assessed. For the present investigation, we applied the following supplementary inclusion criterion: time between fatigue assessment and blood sampling less than 2 months. Patients with alternative diagnoses were excluded from the analyses, as well as patients with comorbidity likely to cause fatigue, other than depression. All patients were followed prospectively and were seen regularly for clinical reassessment. The Ethics Committee of the Erasmus MC University Medical Center Rotterdam approved the study protocol and informed consent was obtained from all participants.

To compare the prevalence and severity of fatigue between patients with CIS and controls, we used data from a group of 113 healthy controls included previously in our centre. ${ }^{12}$ These controls were volunteers recruited from hospital personnel, relatives or friends of patients visiting the outpatient clinic, and volunteers unfamiliar with the study. They had declared themselves to be healthy and not taking any medication that could contribute to fatigue. We also compared the fatigue of patients with CIS with healthy controls and MS patients from the literature. ${ }^{13} 14$

\section{Definitions}

Exacerbation was defined as a worsening of existing symptoms or the appearance of new symptoms 
lasting for more than $24 \mathrm{~h}$, after a period of more than 30 days of improvement or stability, confirmed by neurologic examination. ${ }^{15}$ A temporary neurological deterioration associated with fever was not considered as an exacerbation. All patients in this study were termed CIS, and also included patients who fulfilled the 2010 McDonald criteria. CDMS was diagnosed when there was clinical evidence for dissemination in space and time as described by Poser et al. ${ }^{16}$

\section{Instruments and clinical data}

Fatigue was assessed using Krupp's Fatigue Severity Scale (FSS). ${ }^{17}$ This is a self-administered questionnaire that is widely used and has been validated for use in patients with MS. ${ }^{13} 1417$ It has nine items and seven possible responses per item, ranging from 1 (strong disagreement) to 7 (strong agreement). The mean value of the nine items is the final score. Fatigue is defined as an FSS score of $\geq 5.0$. $^{18-20}$

Since fatigue is known to be associated with depression, we also measured depression, using the Hospital Anxiety and Depression Scale (HADS). The HADS is a self-administered questionnaire consisting of 14 items measuring symptoms of anxiety (7 items) and depression (7 items). ${ }^{21} 22$

Neuroanatomical localisation of CIS was determined at baseline and divided into the following groups: optic nerve, spinal cord, brain stem, other and multiregional.

\section{Measurement of 25-0H-D}

Concentrations of 25-OH-D were determined by a radioimmunoassay method (DiaSorin, USA) using an extraction method. The inter-assay variation coefficient at a concentration of 62 and $109 \mathrm{nmol} / \mathrm{L}$ is $11.6 \%$ and $10.3 \%$, respectively. The intra-assay variation coefficient at the levels is $5.7 \%$ and $6.6 \%$, respectively.

\section{MRI}

All brain MRIs were performed on $1.5 \mathrm{~T}$ scanners with a standard head coil (Philips, Best, the Netherlands, or General Electric, Milwaukee, Wisconsin, USA). Scans typically consisted of an axial T1-weighted sequence, an axial spin echo proton density-weighted and T2-weighted sequence, and an axial fluid-attenuated inversion recovery sequence, with $2-5 \mathrm{~mm}$ images. Postgadolinium T1-weighted sequences were added by the radiologist on indication in patients with T2 lesions suggestive of demyelination. Scans were scored for dissemination in space according to the 2010 McDonald criteria ( $\geq 1$ lesion in at least two of the following areas: periventricular, juxtacortical, infratentorial or spinal cord) ${ }^{23}$ and for gadolinium enhancement. All brain MRIs were performed within 3 months of symptom onset.

\section{Statistical analysis}

Fatigue was analysed both as a continuous variable (FSS) and as a dichotomous variable (yes/no with FSS 5.0 as a cut-off value). Comparison of continuous variables between groups was done using Student $t$ test or ANOVA. Student $t$ test was also used to compare the mean FSS of patients with CIS with values from the literature. Dichotomised variables were compared using the $\chi^{2}$ test. To calculate the association of two continuous variables, correlation analysis was used. The association of fatigue with the diagnosis of CDMS was analysed using survival analysis with univariate and multivariate Cox regression models. In these analyses, 25-OH-D levels were divided into two groups (low: $<50 \mathrm{nmol} / \mathrm{L}$, and high: $>50 \mathrm{nmol} / \mathrm{L}$ ). Anxiety and depression were included in the model as dichotomous variables. All calculations were done using SPSS V.21 for Windows.
Table 1 Baseline characteristics and Fatigue Severity Scale (FSS) outcome for patients with clinically isolated syndrome (CIS) and healthy controls

Patients with $\mathrm{CIS} \mathrm{n}=127 \quad$ Controls $\mathrm{n}=113$

\begin{tabular}{lcc}
\hline Sex (number of females), $\mathrm{n}(\%)$ & $98(77.2 \%)$ & $54(47.8 \%)$ \\
Age (years), mean (SD) & $34.0(8.8)$ & $54.2(14.8)$ \\
Follow-up (months), mean (SD) & $35.4(19.3)$ & \\
FSS, mean (SD) & $4.3(1.9)$ & $2.9(1.1)$ \\
\hline
\end{tabular}

\section{RESULTS}

\section{Participants}

Of the 137 patients in our ongoing CIS study who fulfilled the inclusion criteria, 7 were excluded from the analyses because of alternative diagnoses and 3 were excluded from the analyses because of comorbidity likely to cause fatigue (1 Crohn's disease, 1 untreated hypothyroidism and 1 panhypopituitarism). This left 127 patients for the analyses: baseline characteristics of patients and 113 healthy controls are depicted in table 1. During follow-up, 52 patients (40.9\%) met the criteria for CDMS, with a mean time to diagnosis of 21.1 months (SD 17.0). Blood samples for vitamin D measurement were available for 104 of 127 patients.

\section{Fatigue}

Fifty-nine patients (46.5\%) suffered from fatigue, and the mean FSS for patients with CIS was 4.3 (SD 1.9). In the control group, fatigue was significantly less prevalent $(5.3 \%, \mathrm{p}<0.001)$ and less severe (mean FSS 2.9 (SD 1.1), p<0.001). Similarly, in healthy individuals from the literature, the mean FSS was 3.00 (SD 2.24) ${ }^{13}$ and 3.31 (SD 1.38), ${ }^{14}$ which was significantly lower than in patients with CIS (both $\mathrm{p}<0.001$ ). The FSS of our patients with CIS was similar to that of the MS patients in the cited studies $^{13}{ }^{14}$ (mean 4.66, SD 1.64 and mean 4.81, SD 1.46, both $\mathrm{p}>0.05$ ). FSS scores did not depend on gender or age.

The mean FSS did not differ significantly between patients with different neuroanatomical localisations of CIS. FSS scores did not depend on the time between symptom onset and filling out of the questionnaires.

Fatigue was not associated with 25-OH-D levels: neither with FSS as a continuous variable, nor with fatigue as a dichotomous variable. FSS was not associated with age, nor with MRI measures (number of T2 lesions, gadolinium enhancement, and normal versus abnormal MRI). FSS correlated with both anxiety $(r=0.44, p<0.001)$ and depression $(r=0.51, p<0.001)$.

In univariate analysis, fatigue was associated with a diagnosis of CDMS both as a continuous variable (HR 1.3, 95\% CI 1.1 to 1.6) and as a dichotomous variable (HR 2.6, 95\% CI 1.5 to 4.6). Thirty-two $(61.5 \%)$ of 52 patients who reached CDMS suffered from fatigue, versus 27 (36.0\%) of 75 patients who did not reach CDMS. Patients suffering from fatigue had a shorter time to diagnosis than non-fatigued patients: 32 months in fatigued patients versus 54.8 months in non-fatigued patients (Log Rank test: $\mathrm{p}=0.001)$. The Kaplan-Meier curve of time to CDMS for patients with and without fatigue is shown in figure 1.

\section{5-OH-D}

The mean 25-OH-D concentration of patients with CIS was $67.6 \mathrm{nmol} / \mathrm{L}$ (SD 34.4). 25-OH-D levels showed a seasonal fluctuation with the peak level in July and nadir in January. Thirty-one patients (24.4\%) had low vitamin D levels 


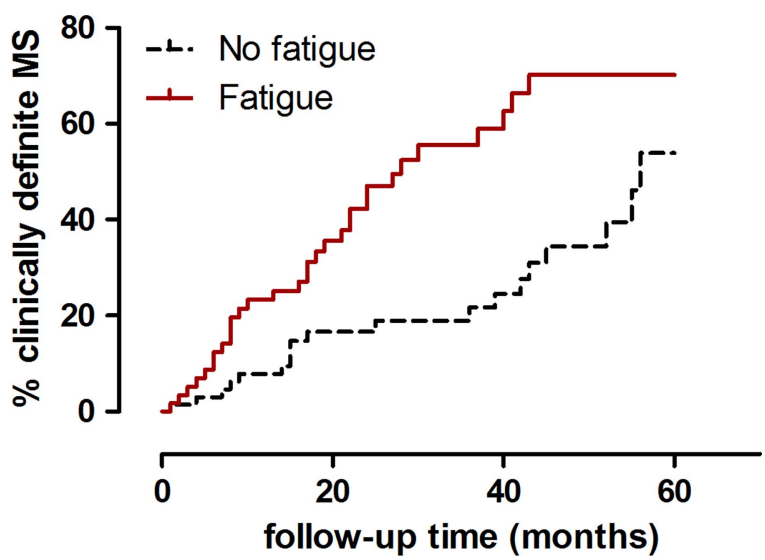

Figure 1 Kaplan-Meier curves for time to clinically definite multiple sclerosis (MS) for patients with and without fatigue (Fatigue Severity Scale $\geq 5.0$ ). Log rank test: $p=0.001$.

$(<50 \mathrm{nmol} / \mathrm{L})$. We found no association between 25-OH-D levels and a diagnosis of CDMS (HR for the low-level group $1.5,95 \%$ CI 0.8 to 2.8 ). Since in a recent study ${ }^{10}$ an increased risk for CDMS was found for only the lowest $10 \%$ of $25-\mathrm{OH}-\mathrm{D}$ concentrations, we also analysed the risk of CDMS for 25-OH-D divided into 10 equal groups, but we did not find an increased risk in the lowest 25-OH-D level group, not even when men and women were analysed separately. 25-OH-D levels were also not associated with time to CDMS, MRI measures and presence of anxiety or depression.

\section{Multivariate analysis}

In a multivariable model correcting for sex, age, localisation of symptoms, vitamin $\mathrm{D}$, anxiety, depression, number of T2 lesions and gadolinium enhancement, the association of fatigue with CDMS was significant, with an HR for CDMS in fatigued patients of 4.5 (95\% CI 1.9 to 10.6). Also in the multivariable model, low 25-OH-D levels were not associated with CDMS (HR $0.9,95 \%$ CI 0.3 to 2.1). In fact, fatigue turned out to be the most significant predictor for CMDS in the model. HRs are shown in table 2 .

\section{DISCUSSION}

In this study, we show that fatigue is a common symptom in patients with CIS, with almost half of patients (46.5\%) suffering from it. The prevalence and the severity of fatigue in patients with CIS are significantly higher than in healthy persons, and similar to the fatigue in MS patients. Importantly, we found that fatigue is associated with a greater risk of CDMS, even independent of MRI measures. The mean time to diagnosis is significantly shorter in patients suffering from fatigue than in non-fatigued patients.

There are several possible explanations for fatigue in patients with CIS. First, it is known that fatigue correlates with neurological disability, ${ }^{24}$ so the fatigue in patients with CIS could be related to the attack itself. However, one would expect this fatigue to differ among patients with different neuroanatomical localisations of CIS (eg, lower FSS in patients with optic neuritis) or lesion loads, and to improve along with amelioration of symptoms; we did not find any association with neuroanatomical localisation, nor with lesion load, nor with time from symptom onset. Second, fatigue could be due to the psychological impact of getting a possible diagnosis of MS. Indeed, we found an association with anxiety and depression, a phenomenon that has been shown previously. ${ }^{25}$ Nevertheless, correction for anxiety and depression in the multiple regression analysis did not influence the association between fatigue and CDMS. Third, fatigue could be related to the second attack, which would explain the association with CDMS. However, as the mean time to the second attack in patients who reach a diagnosis of CDMS is 21.1 months, this does not seem very likely. We

Table 2 HRs for clinically definite multiple sclerosis according to the multivariate analysis

\begin{tabular}{|c|c|c|c|c|c|}
\hline Variable & & $\mathrm{n}$ & Events & HR $(95 \% \mathrm{Cl})$ & p Value \\
\hline Age & $\begin{array}{l}<30 \\
30-40 \\
>40\end{array}$ & $\begin{array}{l}52 \\
40 \\
35\end{array}$ & $\begin{array}{l}21 \\
16 \\
15\end{array}$ & $\begin{array}{l}1 \text { (ref) } \\
0.8(0.3 \text { to } 1.9) \\
0.4(0.1 \text { to } 1.2)\end{array}$ & $\begin{array}{l}-^{*} \\
0.63 \\
0.09\end{array}$ \\
\hline Sex & $\begin{array}{l}\text { Male } \\
\text { Female }\end{array}$ & $\begin{array}{l}29 \\
98\end{array}$ & $\begin{array}{r}8 \\
44\end{array}$ & $\begin{array}{l}1 \text { (ref) } \\
1.3(0.6 \text { to } 3.1)\end{array}$ & $\overline{-}-5$ \\
\hline DIS criteria McDonald 2010 & $\begin{array}{l}\text { Not fulfilled } \\
\text { Fulfilled }\end{array}$ & $\begin{array}{l}45 \\
70\end{array}$ & $\begin{array}{l}12 \\
34\end{array}$ & $\begin{array}{l}1 \text { (ref) } \\
1.7(0.7 \text { to } 4.4)\end{array}$ & $\begin{array}{l}- \\
0.27\end{array}$ \\
\hline Gadolinium enhancement & $\begin{array}{l}\text { No } \\
\text { Yes }\end{array}$ & $\begin{array}{l}45 \\
31\end{array}$ & $\begin{array}{l}16 \\
19\end{array}$ & $\begin{array}{l}1 \text { (ref) } \\
1.8(0.7 \text { to } 5.0)\end{array}$ & $\overline{0} .22$ \\
\hline Vitamin D & $\begin{array}{l}<50 \\
>50\end{array}$ & $\begin{array}{l}31 \\
73\end{array}$ & $\begin{array}{l}16 \\
26\end{array}$ & $\begin{array}{l}0.9(0.3 \text { to } 2.1) \\
1 \text { (ref) }\end{array}$ & $\begin{array}{l}0.75 \\
-\end{array}$ \\
\hline Fatigue & $\begin{array}{l}\text { No } \\
\text { Yes }\end{array}$ & $\begin{array}{l}68 \\
59\end{array}$ & $\begin{array}{l}20 \\
32\end{array}$ & $\begin{array}{l}1 \text { (ref) } \\
4.5(1.9 \text { to } 10.6)\end{array}$ & $\overline{0} .001$ \\
\hline Anxiety & $\begin{array}{l}\text { No } \\
\text { Yes }\end{array}$ & $\begin{array}{l}79 \\
45\end{array}$ & $\begin{array}{l}31 \\
20\end{array}$ & $\begin{array}{l}1 \text { (ref) } \\
0.9(0.4 \text { to } 2.2)\end{array}$ & $\overline{0} .89$ \\
\hline Depression & $\begin{array}{l}\text { No } \\
\text { Yes }\end{array}$ & $\begin{array}{l}97 \\
27\end{array}$ & $\begin{array}{l}39 \\
12\end{array}$ & $\begin{array}{l}1 \text { (ref) } \\
1.7(0.6 \text { to } 5.0)\end{array}$ & $\overline{0}-34$ \\
\hline Symptom localisation & $\begin{array}{l}\text { Optic nerve } \\
\text { Spinal cord } \\
\text { Brain stem } \\
\text { Multiregional } \\
\text { Other }\end{array}$ & $\begin{array}{l}42 \\
35 \\
12 \\
22 \\
15\end{array}$ & $\begin{array}{r}14 \\
13 \\
6 \\
6 \\
11 \\
8\end{array}$ & $\begin{array}{l}1 \text { (ref) } \\
1.0(0.3 \text { to } 3.1) \\
0.4(0.1 \text { to } 1.1) \\
3.2(1.1 \text { to } 9.4) \\
1.3(0.4 \text { to } 5.0)\end{array}$ & $\begin{array}{l}- \\
0.98 \\
0.25 \\
0.03 \\
0.67\end{array}$ \\
\hline
\end{tabular}


favour a fourth explanation: that the fatigue of patients with CIS is the MS-related fatigue presenting itself already at the moment of CIS, independent of the type or severity of CIS. One previous study ${ }^{26}$ has shown that fatigue sometimes precedes other symptoms in MS and another study ${ }^{27}$ described fatigue in early MS; however, in both studies, fatigue was not studied separately in patients with CIS. Although fatigue is very frequently seen in all subtypes of MS, ${ }^{28}$ its pathophysiology is still not well understood. It is likely to be multifactorial, with major roles for inflammatory cytokines associated with the disease combined with CNS dysfunction and secondary mechanisms such as sleep disorders or depression. ${ }^{4}{ }^{28}$ We did not find associations of fatigue with MRI measures. In this study, we also tested the hypothesis that vitamin D is involved in fatigue, but we did not find any evidence for this in patients with CIS. Also, we could not confirm the earlier described association between low vitamin $\mathrm{D}$ concentrations in patients with CIS and risk of CDMS. ${ }^{1029}$ Here, a lack of power cannot be ruled out, but the association, if it exists, will be small as the HR is only 1.2.

Fatigue is a serious disabling symptom of MS, with a negative impact on quality of life. Our study is the first to show that already at the stage of CIS fatigue is very common, with a severity similar to fatigue in MS patients. This fatigue is unrelated to the type or severity of the attack. In addition, we found that fatigue was predictive for definite MS, independent of vitamin $\mathrm{D}$ and known predictors of a subsequent diagnosis of MS such as the McDonald 2010 criteria.

Acknowledgements The authors acknowledge the PROUD participants, Johnny Samijn, MD, PhD, for help with data collection, and Dr Pieter van Doorn, MD, PhD, for help with data collection.

Contributors TFR, NJ and RQH contributed for the study concept and design. TFR, $\mathrm{NJ}$ and DAMS helped in the acquisition of data. TFR, NJ and RQH contributed for the analysis and interpretation of data. TFR contributed to the drafting of the manuscript. All authors contributed to the critical revision of the manuscript for important intellectual content. TFR was involved in Statistical analysis.

Funding This study was not industry-sponsored. All research of ErasMS is supported by the Dutch MS Research Foundation.

Competing interests None.

Ethics approval Ethics Committee of the Erasmus MC University Medical Center Rotterdam.

Provenance and peer review Not commissioned; externally peer reviewed.

\section{REFERENCES}

1 Krupp L. Fatigue is intrinsic to multiple sclerosis (MS) and is the most commonly reported symptom of the disease. Mult Scler 2006;12:367-8.

2 Lerdal A, Celius EG, Krupp L, et al. A prospective study of patterns of fatigue in multiple sclerosis. Eur J Neurol 2007;14:1338-43.

3 Minden SL, Frankel D, Hadden L, et al. The Sonya Slifka Longitudinal Multiple Sclerosis Study: methods and sample characteristics. Mult Scler 2006;12:24-38.
4 Braley TJ, Chervin RD. Fatigue in multiple sclerosis: mechanisms, evaluation, and treatment. Sleep 2010;33:1061-7.

5 Ruiz-Irastorza G, Egurbide MV, Olivares N, et al. Vitamin D deficiency in systemic lupus erythematosus: prevalence, predictors and clinical consequences. Rheumatology (Oxford) 2008;47:920-3.

6 Ruiz-Irastorza G, Gordo S, Olivares N, et al. Changes in vitamin D levels in patients with systemic lupus erythematosus: effects on fatigue, disease activity, and damage. Arthritis Care Res (Hoboken) 2010:62:1160-5.

7 Schnieders J, Willemsen $D$, de Boer $\mathrm{H}$. Factors contributing to chronic fatigue after traumatic brain injury. J Head Trauma Rehabil 2012;27:404-12.

8 Munger KL, Levin LI, Hollis BW, et al. Serum 25-hydroxyvitamin D levels and risk of multiple sclerosis. JAMA 2006;296:2832-8.

9 Runia TF, Hop WC, de Rijke YB, et al. Lower serum vitamin D levels are associated with a higher relapse risk in multiple sclerosis. Neurology 2012;79:261-6.

10 Martinelli V, Dalla Costa G, Colombo B, et al. Vitamin D levels and risk of multiple sclerosis in patients with clinically isolated syndromes. Mult Scler 2014;20:147-55.

11 Zerwekh JE. Blood biomarkers of vitamin D status. Am J Clin Nutr 2008;87:1087S-91S.

12 Merkies IS, Schmitz PI, Samijn JP, et al. Fatigue in immune-mediated polyneuropathies. European Inflammatory Neuropathy Cause and Treatment (INCAT) Group. Neurology 1999;53:1648-54.

13 Valko PO, Bassetti $\mathrm{CL}$, Bloch $\mathrm{KE}$, et al. Validation of the fatigue severity scale in a Swiss cohort. Sleep 2008;31:1601-7.

14 Armutlu K, Korkmaz NC, Keser I, et al. The validity and reliability of the Fatigue Severity Scale in Turkish multiple sclerosis patients. Int J Rehabil Res 2007;30:81-5.

15 Schumacker GA, Beebe G, Kibler RF, et al. Problems of experimental trials of therapy in multiple sclerosis: report by the panel on the evaluation of experimental trials of therapy in multiple sclerosis. Ann N Y Acad Sci 1965;122:552-68.

16 Poser CM, Paty DW, Scheinberg $L$, et al. New diagnostic criteria for multiple sclerosis: guidelines for research protocols. Ann Neurol 1983;13:227-31.

17 Krupp LB, LaRocca NG, Muir-Nash J, et al. The fatigue severity scale. Application to patients with multiple sclerosis and systemic lupus erythematosus. Arch Neurol 1989:46:1121-3.

18 Roelcke U, Kappos L, Lechner-Scott J, et al. Reduced glucose metabolism in the frontal cortex and basal ganglia of multiple sclerosis patients with fatigue: a 18F-fluorodeoxyglucose positron emission tomography study. Neurology 1997:48:1566-71.

19 Bakshi R, Miletich RS, Henschel K, et al. Fatigue in multiple sclerosis: cross-sectiona correlation with brain MRI findings in 71 patients. Neurology 1999:53:1151-3.

20 Lerdal A, Wahl A, Rustoen T, et al. Fatigue in the general population: a translation and test of the psychometric properties of the Norwegian version of the fatigue severity scale. Scand J Public Health 2005;33:123-30.

21 Zigmond AS, Snaith RP. The hospital anxiety and depression scale. Acta Psychiatr Scand 1983;67:361-70.

22 Honarmand $\mathrm{K}$, Feinstein A. Validation of the hospital anxiety and depression scale for use with multiple sclerosis patients. Mult Scler 2009;15:1518-24.

23 Polman $\mathrm{CH}$, Reingold SC, Banwell B, et al. Diagnostic criteria for multiple sclerosis: 2010 revisions to the McDonald criteria. Ann Neurol 2011:69:292-302.

24 Patrick E, Christodoulou C, Krupp LB, et al. Longitudinal correlates of fatigue in multiple sclerosis. Mult Scler 2009;15:258-61.

25 Kroencke DC, Lynch SG, Denney DR. Fatigue in multiple sclerosis: relationship to depression, disability, and disease pattern. Mult Scler 2000;6:131-6.

26 Krupp LB, Alvarez LA, LaRocca NG, et al. Fatigue in multiple sclerosis. Arch Neurol 1988;45:435-7.

27 Simioni S, Ruffieux C, Bruggimann L, et al. Cognition, mood and fatigue in patients in the early stage of multiple sclerosis. Swiss Med Wkly 2007;137:496-501.

28 Krupp LB, Serafin DJ, Christodoulou C. Multiple sclerosis-associated fatigue. Expert Rev Neurother 2010;10:1437-47.

29 Ascherio A, Munger KL, White $\mathrm{R}$, et al. Vitamin $\mathrm{D}$ as an early predictor of multiple sclerosis activity and progression. JAMA Neurol 2014;71:306-14. 